\title{
676 ALTERED CIRCULATING MYELOID STATES ASSOCIATED WITH ANTI-PD-1 RESISTANCE INDUCE T CELL PARALYSIS IN HUMAN BILIARY CANCER
}

Bridget Keenan*, Elizabeth McCarthy, Arielle Ilano, Hai Yang, Li Zhang, Kathryn Allaire, Zenghua Fan, Tony Li, David Lee, Yang Sun, Alexander Cheung, Hewitt Chang, Brenna Sheldon, Robin Kelley, Chun Jimmie Ye, Lawrence Fong. University of California San Francisco, San Francisco, CA, United States

Background Advanced biliary cancers (ABC) have a poor prognosis and low rates of response to immune checkpoint inhibition (CPI), with overall response rates ranging from $3-13 \% .^{1-}$

3 Although suppressive myeloid cells have been proposed as a mechanism of resistance to immunotherapy in general, their relationship to response to CPI is unknown.

Methods We used multiplexed simultaneous single cell RNA sequencing and cell surface proteomics (CITE-seq) to profile circulating immune cells in $\mathrm{ABC}$ patients receiving anti-PD-1 at longitudinal timepoints pre-immunotherapy and on treatment, as well as from healthy donors. We also performed single cell RNA sequencing on resected biliary tumors.

Results We identified a novel population of circulating cancerenriched myeloid cells (CEM) characterized by chemokines and extracellular matrix digestion-related gene expression, which were present pre-treatment. Anti-PD-1 treatment drove the CEMs into two diverging states that were associated with response or resistance to treatment. CEM induced in non-responders constituted over $40 \%$ of the circulating myeloid cells and expressed immunosuppressive programs, including the upregulation of suppressive cytokines and chemokines. The frequency of these myeloid cells were correlated with the abundance of SOCS3-expressing CD4+ T cells. These SOCS3 $+\mathrm{CD} 4+\mathrm{T}$ cells also colocalized with tumor-infiltrating myeloid cells that share CEM gene expression signatures in the biliary cancer microenvironment. Moreover, CEM can directly induce SOCS3-expressing $\mathrm{T}$ cells, which despite their naïve phenotype are functionally unresponsive. Finally, expression signatures of CEM and of SOCS3 + CD $4+\mathrm{T}$ cells are associated with worse survival in a larger cohort of $\mathrm{ABC}$ patients.

Conclusions These results demonstrate the capacity of CEM to induce $\mathrm{T}$ cell paralysis as an alternate mode of tumor-mediated immunosuppression. A deeper understanding of immune cell biology in $\mathrm{ABC}$ provides insights for developing novel therapeutics that can overcome immunotherapy resistance in biliary cancer as well as other tumor types.

Trial Registration NCT02703714

\section{REFERENCES}

1. Ueno $\mathbf{M}$, et al. Nivolumab alone or in combination with cisplatin plus gemcitabine in Japanese patients with unresectable or recurrent biliary tract cancer: a nonrandomised, multicentre, open-label, phase 1 study. Lancet Gastroenterol Hepatol 2019;4:611-621.

2. Piha-Paul SA, et al. Efficacy and safety of pembrolizumab for the treatment of advanced biliary cancer: results from the KEYNOTE-158 and KEYNOTE-028 studies. Int J Cancer 2020.

3. Kim RD, et al. A Phase 2 Multi-institutional study of nivolumab for patients with advanced refractory biliary tract cancer. JAMA Oncol 2020;6:888-894.

Ethics Approval Informed consent was obtained from all patients for participation in the listed trial and for use of blood and tumor samples in research studies.

http://dx.doi.org/10.1136/jitc-2021-SITC2021.676 